\title{
Epidemiology of burns at the University Teaching Hospital of Kigali, Rwanda
}

\author{
Authors: J. P. Dushime ${ }^{1, *}$; L. Jing ${ }^{2}$; D. Uwamahoro ${ }^{1}$; G. Mbanjumucyo ${ }^{3}$;. Tepp ${ }^{4,5}$; P. Shama ${ }^{4,5}$ \\ Affiliations: ${ }^{1}$ University of Rwanda, Kigali, Rwanda; ${ }^{2}$ Case Western Reserve University School \\ of Medicine, USA; ${ }^{3}$ King's College hospital, London, UK; ${ }^{4}$ New York Presbyterian hospital, USA; \\ ${ }^{5}$ Columbia University School of Medicine, USA
}

\begin{abstract}
INTRODUCTION: Burns are the fourth most common type of injury presenting to the emergency department in Rwanda. However, there is little data on the epidemiology of burn patients, which is needed to inform public health measures for burn prevention.

This study aimed to describe the characteristics and outcomes of burn patients presenting to the Emergency of the University Teaching Hospital Kigali.

METHODS: This single-center prospective study evaluated patients with burn injuries presenting at CHUK emergency between 1 June and 31 December 2019. Data on demographics, burn characteristics, burn management, disposition and patient outcomes were collected, stored in Excel and analyzed descriptively using SPSS 24.

RESULTS: Of the 96 patients enrolled; (58.3\%) were male. The median age was 32.7 years. Most patients were pediatric (70.9\%), with one to five-year-olds being the most affected age group. Scalding was the most common cause of burn at (71.9\%). Most burns were accidental (95.8\%) and superficial dermal in degree (72.9\%). The mean time between injury and presentation was 18.1 hours. There were 12 mortalities (12.5\%) within the 30 days post-injury, with scald injuries as the most frequent cause of mortality. Higher mortality was significantly associated with increased body surface area affected $(p=0.001)$.

CONCLUSION: Children between the ages of one and five years are the most affected by burns and scalding is the most common burn etiology. Public health measures should aim to prevent burn injuries in the pediatric population. This study was approved by the University of Rwanda / CMHS Institutional Review Board (IRB) No 136/CMHS IRB/ 2019.
\end{abstract}

Keywords: Burns, Rwanda, Emergency Service, Hospital, Public Health

\section{INTRODUCTION}

The World Health Organization (WHO) broadly defines a burn as an injury caused by heat (hot objects, gases, or flames), chemicals, electricity and lightning, friction, or radiation [1]. Globally, approximately 11 million people sustain burns requiring medical attention each year [1]. Burns

*Corresponding author: Jean Paul DUSHIME, Department of Emergency Medicine, University of Rwanda Kigali, Rwanda, Email: dusjpaul4@gmail.com, Telephone: + 250783197515; Potential Conflicts of Interest (Col): All authors: no potential conflicts of interest disclosed; Funding: All authors: Our study used a small budget from the principal investigator. There was no external sponsors; Academic Integrity. All authors confirm that they have made substantial academic contributions to this manuscript as defined by the ICMJE; Ethics of human subject participation: The study was approved by the local Institutional Review Board. Informed consent was sought and gained where applicable; Originality: All authors: this manuscript is original has not been published elsewhere; Review: This manuscript was peer-reviewed by three reviewers in a double-blind review process; Type-editor:Himani (USA).

Received: $28^{\text {th }}$ August 2020; Initial decision given: $25^{\text {th }}$ July 2021; Revised manuscript received: $03^{\text {th }}$ August 2021; Accepted: $23^{\text {th }}$ November 2021. Copyright: (C) The Author(s). This is an Open Access article distributed under the terms of the Creative Commons Attribution License (CC BY-NC-ND) (click here) which permits unrestricted use, distribution, and reproduction in any medium, provided the original work is properly cited. Publisher: Rwanda Biomedical Centre (RBC)/Rwanda Health Communication Center, P. O. Box 4586, Kigali. ISSN: 2079-097X (print); 2410-8626 (online) 
are the fourth most common substantial injury after road traffic accidents, falls, and interpersonal violence [2]. Fire-related burns alone cause more than 300,000 deaths each year [3]. The populations most affected by burns are children and the elderly [4].

More than $90 \%$ of burn injuries occur in low- and middle-income countries (LMICs) [1]. In developing countries, $95 \%$ of burn-related deaths can be attributed to poor access to primary care [5]. The appropriate acute management of burn injuries in the emergency department (ED) is critical to preventing physical disability and psychological complications for patients. In East African countries such as Uganda and Tanzania, studies on the epidemiology of burn injuries have found that the age range was $1-10$ years (64.3\%), 53\% were female. In contrast, $47 \%$ were male, and the scalding burns formed $65 \%$ of all burn injuries $[6,7]$. The management of burn injuries is significantly costly and has impacts on the economy, health systems and quality of life of victims [8].

In Rwanda, most burn patients are treated at emergency departments of district hospitals [9], while patients with severe burns requiring advanced care are transferred to the emergency department of the University Teaching Hospital Kigali (CHUK) [2]. Accurate epidemiological data on burns can inform appropriate public health measures for avoiding burns and improve management at the emergency department [10]. Prevention strategies need to be tailored to specific environments, taking into account local risk factors and available resources [9].

This study aimed to describe the characteristics and outcomes of burn patients presenting to the emergency department at a major hospital in Kigali, Rwanda. The research objectives were to identify patient demographic information, determine burn characteristics, describe the burn management at the CHUK ED, to determine patient outcomes 30 days post-injury.

\section{METHODS}

Study design: This single-center, prospective study evaluated patients with burn injuries arriving at the CHUK ED between 1 June and 31 December 2019.

CHUK, an urban referral and tertiary-care teaching hospital that is the only public tertiary hospital in Kigali and has approximately 560 inpatient and 24
ED beds. The hospital has a high volume of burn patients. The ED is covered by general practitioners (GPs), emergency medicine (EM) residents, and emergency specialists.

Participants: Our study population consisted of all burn patients who presented to the ED during the defined study period. The inclusion criteria were all patients with burn injuries presenting to the CHUK ED during the study period. The exclusion criteria were patients who refused to sign the informed consent and patients who were unable to give consent due to incapacitation by their injuries and had no family member present to provide consent. The primary outcome was to identify patient demographic information (age, sex, district) and burn characteristics (etiology, circumstance, timing from injury, degree of burn, total body surface area). The Secondary outcomes were to describe the burn management at the CHUK ED (Fluid resuscitation, painkillers, debridement, disposition and length of stay in ED) and to determine patient outcomes 30 days post-injury.

Data sources: Data were collected by interviews and some information from the charts. A data collection form was developed by assessing the relevant literature. The form was assessed for content validity to ensure that it was acceptable. Data were collected by the principal investigator and three research assistants (nurses) alternating for a shift of 12 hours for 24 hours a day, seven days a week. They ensured that every patient with burn was prospective enrolled in the study. After burns, patients were treated by the emergency team, they were approached and explained about the study if he/she accepted the participation in the study, the consent form was signed, then data collected using a data collection tool for the outcome 30 days post-injury were assessed by review the medical chart for the one still in the hospital or by phone call for the patient already discharged home. No randomization was used; this study was purely observational.

A sample size of ninety patients was determined to be necessary for this study. The sample size was calculated using the Raosoft sample size calculator. The calculation was based on $50 \%$ response distribution, $5 \%$ margin of error and 95 $\%$ confidence interval.

Variables: Data variables collected included: age, sex, district, etiology of burn, circumstances of burn, TBSA affected, degree of burn, length of ED stay, and outcomes 30 days post-injury. 
The classification of different variables in some groups was done according to relevant literature. The age was classified in 8 age grou ps $(<1,1-5,6-15,16-25,26-35,36-45,46-65,>65$ years) and into three categories as pediatrics (015 years), Adult (16-64 years) and elderly (>65 years). In terms of etiology, burns injuries have been classified to be caused by scalds, electricity, fire (flame), explosion, and chemical burns. The circumstances of burn were: accident, assault and suicide. The degrees of burns are divided into 4 groups: epidermal, superficial dermal, deep dermal and full thickness. The TBSA was classified in 4 groups: $1-10 \%, 11-30 \%, 31-60 \%, 61-100 \%$.

Statistical methods: Data were entered into Excel and analyzed using SPSS24 software. Descriptive statistics with frequency and percentages for categorical data were calculated. Taking the outcome "death" as a dependent variable, all the independent variables were analyzed and statistical significance set as a p-value less than 0.05 at 95\% confidence interval. Pearson chi-square test was used to analyze factors associated with mortality.

Ethical approval: This study was approved by the CMHS Institutional Review Board (IRB) No 136/ CMHS IRB/ 2019 and the CHUK ethics committee EC/CHUK/0108/2019. There was no risk to participate in our study and no Incentives given to the participant. The consent form was signed before participation in the study and ensured confidentiality. The manuscript was prepared based on the STROBE checklist for observational studies.

\section{RESULTS}

A total of 3,856 patients presented to CHUK from June 2019 to December 2019 and 2,152 patients were trauma patients with 98 patients with burn injuries. Two patients refused to provide consent, and a total of 96 were enrolled in the study.

More than half of the patients were male (58.3\%), and the median age was 32.7 years (Table 1 ).

The most affected population was children (70.9\%), with one to five years being the most affected age group (Figure 1). Most patients were from Kigali City (70.9\%) and all provinces in Rwanda were represented (Table 1). Most patients were received from other hospitals (65.6\%), and (47.9\%) arrived at CHUK via an ambulance compared to (52.1\%) who arrived via private transport (Table 1). The mean time from the burn injury to the presentation at the ED was 18.1 hours.

The most common burn etiology was scalding (71.9\%), followed by explosion (13.5\%), with chemical burns being the least common

Table 1: Demographic information of burn patients presenting at CHUK Emergency Department

\begin{tabular}{lrrr}
\hline & & $\mathbf{n}$ & $\mathbf{\%}$ \\
\hline Sex & Male & 56 & $58.3 \%$ \\
Age (Mean 10.3years) & Female & 40 & $41.7 \%$ \\
& Paediatric (0-15years) & 68 & $70.9 \%$ \\
& Adult (16-64years) & 26 & $27.1 \%$ \\
Province of Residence & Elderly (>65years) & 2 & $2.0 \%$ \\
& Kigali & 68 & $70.9 \%$ \\
Received form & South & 11 & $11.5 \%$ \\
Mode of arrival & North & 9 & $9.4 \%$ \\
& West & 6 & $6.2 \%$ \\
& East & 2 & $2.0 \%$ \\
& Other hospitals & 63 & $65.6 \%$ \\
& Home & 33 & $34.4 \%$ \\
& Ambulance & 46 & $47.9 \%$ \\
\hline
\end{tabular}




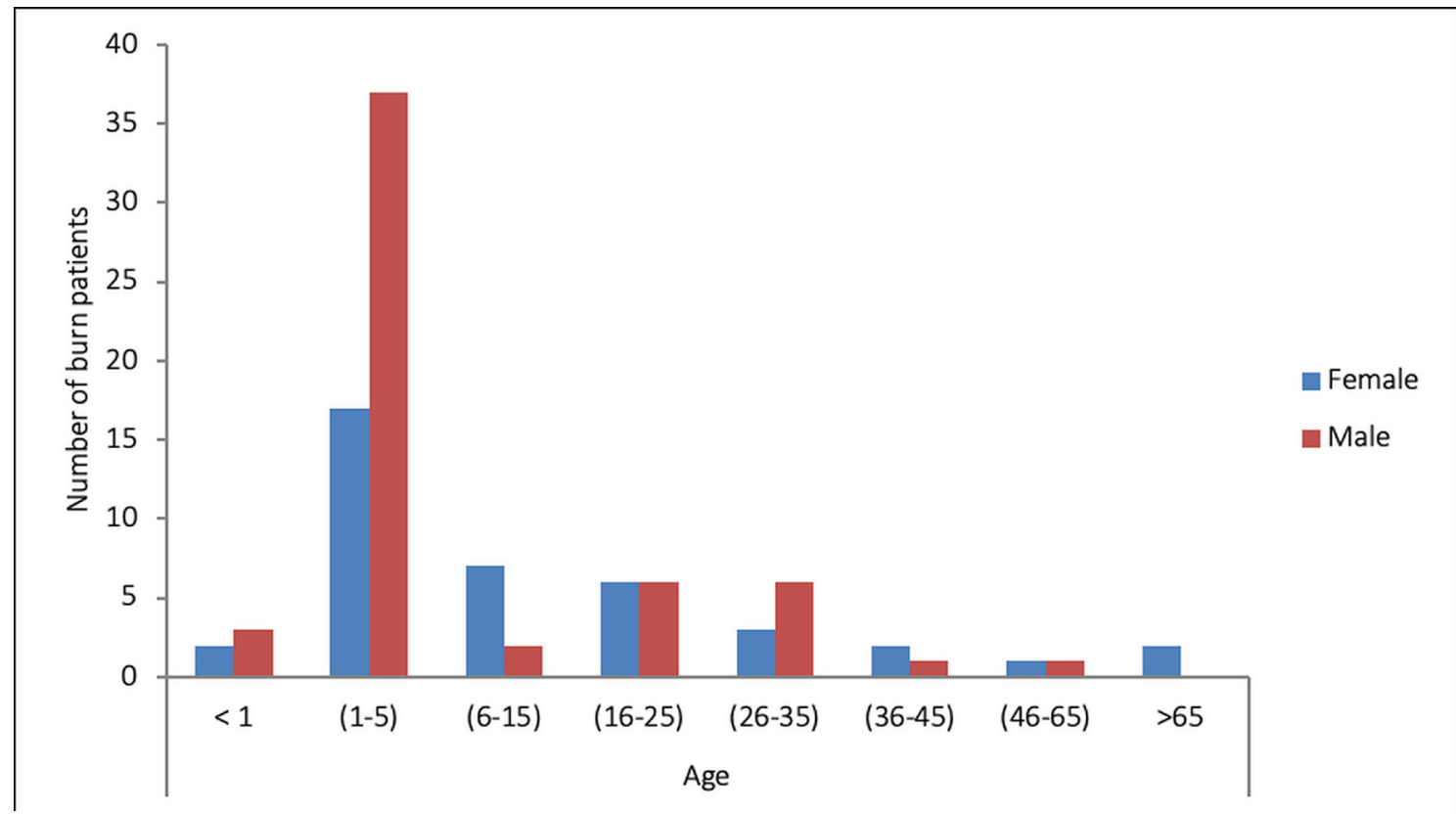

Figure 1: Burn patients presenting at CHUK Emergency Department by age and sex

mechanism (1\%) (Table 2). The vast majority of burns were accidental (95.8\%) (Table 2). The most common degree of burn observed was superficial dermal (72.9\%) and the predominant range of total body surface area (TBSA) burned was 1-10\% (43.8\%) (Table 2).

Regarding management in the emergency department, all patients' wounds were debrided and dressed before discharge or admission to the hospital. Most patients received analgesics (70.8\%) and fluid resuscitation (63.5\%), while a fraction received tetanus immunoglobulin (TIG) (17.7\%) and only few patients underwent advanced airway management by intubation (6.3\%) (Table 2). More than half of patients (53.1\%) were admitted to the hospital, with $44.8 \%$ in the burn unit and $8.3 \%$ in the intensive care unit (ICU). Additionally, $46.9 \%$ of patients were discharged home from the ED (They had remained in ED for lack of bed in other wards) (Table 2).

The mean length of stay in the ED was 22.5 hours. There were 12 deaths (12.5\%) in the 30-day period after injury, with most of the mortality occurring among male patients who formed seven out of the 12 deaths (Table 2). Scald injuries were seen in 50\% of the deaths (Table 2). Mortality was significantly associated with TBSA $(p=0.001)$ and full-thickness burns $(p=0.01)$ (Table 2$)$.

\section{DISCUSSION}

The most affected population was children, with one to five years being the most affected age group (Figure 1). The most common burn etiology was scalding (71.9\%) (Table 2). The most common degree of burn observed was superficial dermal $(72.9 \%)$ and the predominant range of total body surface area (TBSA) burned was 1-10\% (43.8\%) (Table 2). Regarding management in the ED, most patients were debrided, received analgesics and fluid resuscitation, while a few patients received tetanus immunoglobulin (TIG) (Table 2). More than half of patients (53.1\%) were admitted to the hospital. There were 12 deaths (12.5\%) in the 30day period after injury.

This study of 96 burn patients at the CHUK ED showed that the most affected by burns were pediatric patients, and the age group most affected were children between the ages of one and five years old. These findings are similar to those of a study in Uganda [9]. The high incidence in this age group may be explained by the fact that children at this age, want to learn new things, have decreased perception of risk, decreased coordination, and are closer to the ground where splashes and spills most frequently occur [11]. Preventive measures should be taken to prevent these injuries, such as close supervision of children to decrease exposure to burn risks. Scalding was the most common burn 
Table 2: Characteristics and outcomes of burn patients presenting at the CHUK Emergency Department

\begin{tabular}{|c|c|c|c|c|c|c|c|c|}
\hline & & & cal outco & & & Total & & P-value \\
\hline & & & Recover & & Death & & & \\
\hline & & $\mathbf{n}$ & $\%$ & $\mathbf{n}$ & $\%$ & n & $\%$ & \\
\hline Sex & Male & 49 & $51.0 \%$ & 7 & $7.2 \%$ & 56 & $58.3 \%$ & 0.35 \\
\hline & Female & 35 & $36.5 \%$ & 5 & $5.2 \%$ & 40 & $41.7 \%$ & \\
\hline Age Category & Pediatric & 63 & $65.6 \%$ & 5 & $5.2 \%$ & 68 & $70.8 \%$ & 0.24 \\
\hline & Adult & 20 & $20.9 \%$ & 6 & $6.2 \%$ & 26 & $27.1 \%$ & \\
\hline & Elderly & 1 & $1.0 \%$ & 1 & $1.0 \%$ & 2 & $2.0 \%$ & \\
\hline Etiology of burn & Scald & 63 & $65.6 \%$ & 6 & $6.2 \%$ & 69 & $71.9 \%$ & 0.25 \\
\hline & Explosion & 10 & $10.4 \%$ & 3 & $3.1 \%$ & 13 & $13.5 \%$ & \\
\hline & Fire & 7 & $7.3 \%$ & 3 & $3.1 \%$ & 10 & $10.4 \%$ & \\
\hline & Electrical & 3 & $3.1 \%$ & 0 & $0.0 \%$ & 3 & $3.1 \%$ & \\
\hline & Chemical & 1 & $1.0 \%$ & 0 & $0.0 \%$ & 1 & $1.0 \%$ & \\
\hline Degree of burn & Epidermal & 11 & $11.5 \%$ & 0 & $0.0 \%$ & 11 & $11.5 \%$ & 0.01 \\
\hline & Superficial dermal & 62 & $64.6 \%$ & 8 & $8.3 \%$ & 70 & $72.9 \%$ & \\
\hline & Deep dermal & 11 & $11.5 \%$ & 2 & $2.0 \%$ & 13 & $13.5 \%$ & \\
\hline & Full thickness & 0 & $0.0 \%$ & 2 & $2.0 \%$ & 2 & $2.0 \%$ & \\
\hline Total body surface area & $1-10 \%$ & 42 & $43.7 \%$ & 0 & $0.0 \%$ & 42 & $43.7 \%$ & 0.001 \\
\hline & $11-30 \%$ & 32 & $33.3 \%$ & 2 & $2.0 \%$ & 34 & $35.4 \%$ & \\
\hline & $31-60 \%$ & 10 & $10.4 \%$ & 5 & $5.2 \%$ & 15 & $15.6 \%$ & \\
\hline & $61-100 \%$ & 0 & $0.0 \%$ & 5 & $5.2 \%$ & 5 & $5.2 \%$ & \\
\hline Disposition & Burn unit & 36 & $37.5 \%$ & 7 & $7.2 \%$ & 43 & $44.8 \%$ & 0.001 \\
\hline & Intensive care unit & 3 & $3.1 \%$ & 5 & $5.2 \%$ & 8 & $8.3 \%$ & \\
\hline & Discharge home from ED* & 45 & $46.9 \%$ & 0 & $0.0 \%$ & 45 & $46.9 \%$ & \\
\hline
\end{tabular}

*They had remained in ED for lack of bed in other wards ED: Emergency Department

mechanism in children and these injuries tended to result from the accidental overturning of a boiled fluid. For adults, these injuries resulted from accidental explosions of flammable substances such as gas, as most Rwandans have begun to use gas in cooking.

The mean time between injury and presentation was 18.1 hours, as most patients who presented at CHUK for burn injuries were transferred from the district hospital. Regarding management performed at the emergency department, all patients were debrided and dressed before admission or discharge from the hospital. The majority of patients received analgesics and fluid resuscitation, and few received tetanus immunoglobulin (TIG). Collaboration with ED staff is needed to improve management so that every burn patient receives fluids, analgesics, and TIG if necessary. Just over one-half of patients were admitted to the hospital, mainly to the burn unit. The mortality after 30 days was $12.5 \%$, a rate similar to that found in Tanzania, Uganda and Nigeria $[6,7,8]$.

A limitation of this study is that it may not be generalizable to the broader population of Rwanda, as it was a single-centre study focusing on patients presenting at a tertiary-level facility. We expect that a significant number of burns do not present to the hospital and that many are treated prior to transfer at the clinic and district level. Thus, this study may overestimate the severity of burn injuries. Furthermore, the sample size was small, which reduced the power of our study. Finally, many patients were transferred from 
district hospitals after preliminary stabilization, or treatment. Future research on burn injuries should aim to understand the broader epidemiology of burns in Rwandan patients. A multi-centre study would provide more complete data to guide health education and interventions.

\section{CONCLUSION}

Children between one and five years of age are

\section{REFERENCES}

[1] M. M. Rybarczyk et al., "A systematic review of burn injuries in low- and middle-income countries: Epidemiology in the WHO-defined African Region," African J. Emerg. Med., vol. 7, no. 1, pp. 30-37, 2017.

[2] WHO, "Management of Burns and Scalds," WHO Surg. Care Dist. Hosp., pp. 1-7, 2003.

[3] N. Aksoy, S. Arli, and O. Yigit, "A Retrospective Analysis of the Burn Injury Patients Records in the Emergency Department, an Epidemiologic Study," Emergency, vol. 2, no. 3, pp. 115-120, 2014.

[4] E. Broadis, T. Chokotho, and E. Borgstein, "Pediatric burn and scald management in a low resource setting: A reference guide and review," African J. Emerg. Med., vol. 7, pp. S27-S31, 2017. [5] A. Landry, H. Geduld, A. Koyfman, and M. Foran, "An overview of acute burn management in the Emergency Centre," African J. Emerg. Med., vol. 3, no. 1, pp. 22-29, 2013.

[6] D. L. Kitara, J. Aloyo, J. H. Obol, and D. A. Anywar, "Epidemiology of burn injuries: A basis for prevention in a post-conflict, Gulu, northern the age group most affected by burns in our study, and the most common cause of burns was scald injuries occurring at home. The hospital mortality is increased when burns exceed 30\% TBSA. Public health measures should focus primarily on preventing burn injuries in the pediatric population. Community education on kitchen and cooking safety could positively impact the prevalence and outcomes of both pediatric and adult burn injuries.

Uganda: A cross- sectional descriptive study design," J. Med. Med. Sci., vol. 2, no. July, pp. 990996, 2011.

[7] A. H. Outwater, H. Ismail, L. Mgalilwa, M. Justin Temu, and N. A. Mbembati, "Burns in Tanzania: morbidity and mortality, causes and risk factors: a review.," Int. J. Burns Trauma, vol. 3, no. 1, pp. 18-29, 2013.

[8] C. E. Okafor, O. Onunka, and L. N. Idoko, "Cost-utility of burns management in Nigeria: a case study of the National Orthopedic Hospital, Enugu.," Ann. Burns Fire Disasters, vol. 30, no. 1, pp. 9-12, 2017.

[9] R. T. . Petroze et al., "Patterns of injury at two university teaching hospitals in Rwanda: Baseline injury epidemiology using the rwanda injury registry," Rwanda Med. J., vol. 71, no. 1, pp. 5-8, 2014.

[10] T. Nottingham and N. E. User, "Othman , Nasih ( 2010 ) Epidemiology of burn injuries in Sulaymaniyah province of Iraq. PhD thesis, University of Nottingham," 2010.

[11] D. J. Van Hoving, B. K. Barnetson, and L. A. Wallis, "Emergency care research priorities in South Africa," South African Med. J., vol. 105, no. 3, pp. 202-208, 2015. 